\title{
Preference Aggregation and Repeat Buying in Households
}

\author{
SUNIL GUPTA* \\ Graduate School of Business, University of Michigan, Ann Arbor, MI 48109-1234
}

JOEL H. STECKEL

Stern School of Business, New York University, New York, NY 10012

Key words: Brand switching analyses, Markov transition matrices, Houschold-level panel data, Individual-level transition matrices, variety seeking, family decision making

\begin{abstract}
Marketing researchers have long used brand switching analyses and Markov transition matrices to gain insights into managerial problems. Almost without exception, this work makes (inappropriate) inferences about individual consumers by analyzing household-level data. This paper presents a procedure based on the distribution of run lengths in household level panel data that allows more insights into the choice behavior of the individuals in the household. We test these procedures in a large simulation study by attempting to recover the underlying (known) structure of the process generating a string of panel data. Finally, we use the procedure to classify the purchase behavior, with respect to powdered soft drinks, of a set of households in a panel. Our results show that marketing scientists have the potential to learn and test more hypotheses about the individuals in a household by examining the distribution of run lengths.
\end{abstract}

Marketing researchers have long suggested that brand switching analyses can provide useful insights into such managerial problems as promotion assessment and market structure analysis (Lehmann 1972; Kalwani and Morrison 1977). Implicitly, these approaches assume that the essence of the underlying behavior is captured by some specified Markov process. Early work focussed on learning what we might be able to say about households from aggregated market level brand switching data (Frank 1962; Massy, Montgomery, and Morrison 1970). Recent advances in scanner panels have made the household a more accessible unit of analysis. However, as Kahn, Morrison and Wright (KMW) (1986, p. 261) note, "almost all of the recent marketing literature on variety seeking and reinforcement behavior uses household level data, yet makes individual (our emphasis) consumer level inferences." This led KMW to study household level Markov transition matrices from the perspective of aggregating individual household members. They conclude that, under certain " "standard' assumptions, aggregating individuals to households always makes the household behavior more zero-order than the typical individual in the household (p. 261)."

*We gratefully acknowledge Professor Bari Harlam of the University of Rhode Island for providing the panel data. 
Currently, no procedures exist for disentangling household level transition matrices to gain insight about the individual household members. An essential premise of this paper is that household level panel data contain information about the individuals which is not captured by the corresponding Markov transition matrices. Indeed, Bass et al. (1984) and Jeuland, Bass, and Wright (1980), among others, performed tests based on the total number of runs of consecutive purchases in a given string of panel data.

We contend that even more information is available. While previous research concentrates on the total number of runs, we focus on the distribution of their lengths. We propose a test, based on the distribution of run lengths in household level panel data, which lends insight into the individual level transition matrices and the process by which they are combined to produce the observed string of purchases. Such insight provides a more accurate representation of the consumer behavior underlying a string of panel data.

\section{Conceptualization}

For simplicity and ease of exposition, assume a two-person household participating in a two-brand market. The people are designated $I$ and 2 and the brands are designated $A$ and $B$. We assume that each individual's preferences are captured by his/her transition matrix.

$$
\boldsymbol{P}_{i}=\left(\begin{array}{ll}
p_{A A} & p_{A B} \\
p_{B A} & p_{B B}
\end{array}\right)
$$

where $p_{q r}$ is the probability of buying brand $r$ if brand $q$ was bought on the previous purchase occasion. This general first-order transition matrix can reflect a variety of individual preference structures:

$\begin{array}{lll}\text { i) } & \text { strict preference for } A(B) & p_{A A}\left(p_{B B}\right)>0.5 \text { and } p_{B A}\left(p_{A B}\right)>0.5 \\ \text { ii) } & \text { variety seeking } & \left.p_{A A}<0.5 \text { and } p_{B B}<0.5\right) \text {, and } \\ \text { iii) } & \text { reinforcement } & p_{A A}>0.5 \text { and } p_{B B}>0.5 .\end{array}$

Furthermore, if $p_{A A}=p_{B A}$ and $p_{A B}=p_{B B}$, the behavior is zero order.

When $\boldsymbol{P}_{1} \neq \boldsymbol{P}_{2}$, the resulting preference conflict among individuals may be resolved at the household level in a variety of ways. In general, following much of the group decision making literature (Corfman and Gupta 1993; Steckel et al. 1991 for reviews), we assume that

$$
\boldsymbol{P}_{H}=w_{1} \boldsymbol{P}_{1}+w_{2} \boldsymbol{P}_{2}
$$

Because the $w_{i}$ 's represent the relative influence of each individual's preferences, equation $I$ represents a summary of the household's aggregation mecha- 
nism (AM). For example, if $w_{1}=1$ and $w_{2}=0, P_{H}=P_{1}$, and the household's choices are completely determined by the first individual's transition matrix. Less extreme weights would imply a household transition matrix which was some weighted average of the individual matrices. There are, of course, many possible ways in which the weights in equation I may be specified, each representing a different AM. We focus on three prominent possibilities:

i) Strict Compromise. Under this AM, $w_{1}=w_{2}=0.5$, and the household members behave as a single unit, compromising on each purchase occasion. The household's transition matrix is the average of the individual matrices; $i . e_{.}, P_{H}$ $=0.5 \boldsymbol{P}_{1}+0.5 \boldsymbol{P}_{2}$. Because the choice alternatives are discrete, when both members consider the choice to be important, they may compromise on each purchase occasion through a simple averaging of the individual purchase probabilities. Note, by construction, that such a household's behavior is indistinguishable from that of a single individual with transition matrix $\boldsymbol{P}_{H}$.

ii) Turn-Taking. Recent research suggests that groups often resolve conflicts by equalizing the gains/losses of their members over time (Corfman and Gupta 1993; Corfman and Lehmann 1987). Turn-taking is one way to accomplish this. For example, some studies have found that groups will explicitly take turns with respect to jointly consumed items such as food and entertainment (Corfman, Lehmann, and Steckel 1990; Steckel, Lehmann, and Corfman 1988). That is, the $w_{i}$ 's change predictably from purchase occasion to purchase occasion $\left(\boldsymbol{P}_{\boldsymbol{H}}=\boldsymbol{I} \cdot \boldsymbol{P}_{1}+0 \cdot \boldsymbol{P}_{2}\right.$ on occasion $1, \boldsymbol{P}_{H}=0 \cdot \boldsymbol{P}_{1}+1 \cdot \boldsymbol{P}_{2}$ on occasion 2 , and so on $)$. Under this AM, the person whose turn it is to choose at time $t$ will examine what was chosen for the household at $t-l$ (by the other member) and make the decision based on his/her own transition matrix. The household's purchase string, therefore, results from the alternating application of two separate transition matrices.

iii) Decoupled-superpositioning. This AM is suggested by $\mathrm{KMW}$. Unlike the previous two AM's, this process does not constrain the household to make a single joint choice. Instead, each individual purchases according to his or her own transition matrix, as and when the need arises. The superpositioning of these two individual purchase strings is the observed household level panel data. This may be the case for a product category such as coffee, where consumption is not joint and the family budget permits each member to satisfy his/ her own preferences. Because the individual interpurchase times are assumed to be independent, identical and exponentially distributed (see KMW), the household behaves as if a given individual's weight (say $w_{1}$ ) is 1 on some purchase occasions and 0 on others. However, unlike the predictable weight changes under turn-taking, $w_{1}$ is 1 or 0 depending on the exponentially distributed interpurchase times.

These are certainly not the only AM's that can exist. Many others which are a mixture or modifications of these are possible. For example, each individual could 
buy for him/herself, but the two could take turns going to the market. This would be an instance of decoupled superpositioning with deterministic interpurchase times, and the resulting purchase string would have runs of length 1 and 3 only. ${ }^{1}$

For pedagogical reasons, however, we restrict our attention to the three extreme cases. The strict compromise mechanism is different from the other two in that it represents a single mechanism (transition matrix). It is indistinguishable from a single individual, and it is the only one which does not involve household members purchasing separately. The other two represent endpoints on a continuum. First, under turn-taking each individual makes the current decision based on the household's previous purchase. Under decoupled-superpositioning, each individual's current choice depends on his/her own previous choice. Second, decoupled-superpositioning has the individuals purchasing at exponential intervals. Given the memoryless property of the exponential distribution, there is no way to know which individual is going to purchase next. If the exponential parameters are equal, the probability for each is 0.5 . In a very real sense then, this represents the most random process. In contrast turn-taking represents the least random process. If we know who purchased last, we definitely know who will purchase next.

In addition, even though these AM's may characterize only a minority of households, their modifications may produce behaviors which resemble the original týpes. However, many of these modifications may produce behaviors which resemble the pure types. For example, if one household member makes every tenth decision (rather than every other one), or if the compromise weights are 0.1 and 0.9 (instead of 0.5 and 0.5 ) the household's behavior will be almost identical to that of the dominant individual. Our results, then, provide limits on the extent to which intuitively appealing deviations from the pure cases can be discriminated, without having to collect more information about the household.

\section{A motivating example}

Consider a household consisting of a pure reinforcer and a pure variety seeker, i.e., $p_{1}=\left(\begin{array}{ll}1 & 0 \\ 0 & 1\end{array}\right)$ and $p_{1}=\left(\begin{array}{ll}0 & 1 \\ 1 & 0\end{array}\right)$. Person $l$ always stays with the last brand bought and person 2 always switches. Next, suppose that the household's preferred AM is turn-taking. Now, assume both that $A$ was the last brand bought and that it is person l's turn. Being a pure reinforcer (s)he chooses $A$. It is now person 2 's turn. Being a pure variety seeker, (s)he chooses $B$. Next, person $I$ reinforces with $B$, person 2 switches to $A$, person $I$ stays with $A$, person 2 switches to $B$, and so on. Continuing in this manner results in the following string of purchases in the household's panel data:

$A A B B A A B B A A B B A A B B A A B B A A B B A A B B A A B B \ldots$

The resulting household transition matrix is $p_{H}=\left(\begin{array}{cc}0.5 & 0.5 \\ 0.5 & 0.5\end{array}\right)$, which is the 
same as we would expect from a brand choice process governed by a simple coin toss (or, purchasing with a strict compromise matrix), or even decoupled-superpositioning. Note that the total number of runs in the string is one-half the length of the string. This is the expected number in the coin-toss model. Thus, looking at the total number of runs provides no useful additional information for recovering the underlying behavior of the household. But the turn-taking process described above is very systematic. Each run is of length 2 . There is no variance! This presents strong evidence against the coin-toss process.

This example is certainly extreme. Nevertheless, three important ideas emerge from it. First, all identical household level transition matrices are not equivalent. Vastly different processes can produce the same household transition matrix. Second, both the individual preferences (captured by the respective transition matrices) and the AM must be understood for proper classification of the household process. We have already shown that given $\boldsymbol{P}_{1}$ and $\boldsymbol{P}_{2}$, any of the three AM's can result in the same household transition matrix, $\boldsymbol{P}_{H}$. It is also clear that given a AM many different $\boldsymbol{P}_{i}$ 's can result in the same $\boldsymbol{P}_{\boldsymbol{H}}$ (e.g., $\boldsymbol{p}_{1}=\left(\begin{array}{cc}0.70 & 0.30 \\ 0.30 & 0.70\end{array}\right)$ and $\boldsymbol{p}_{2}=$ $\left(\begin{array}{ll}0.30 & 0.70 \\ 0.70 & 0.30\end{array}\right)$ would also result in $p_{H}=\left(\begin{array}{ll}0.50 & 0.50 \\ 0.50 & 0.50\end{array}\right)$ for each of the AM's).

Consequently, relying only on the household transition matrix, or the total number of runs can lead to gross misclassification. Finally, and most importantly, the example suggests that the distribution of run lengths in the panel string can be helpful in determining the underlying processes. We now need to examine whether it is possible to construct a test based on the distribution of run lengths which, given the household's purchase string, can reliably recover $\boldsymbol{P}_{1}, \boldsymbol{P}_{2}$ and the AM.

\section{The run length test}

The test we propose classifies a string of panel data according to which underlying behavior or model it resembles most closely. It consists of four steps.

Step 1: Construct a library of hypothesized behaviors.

A complete library of possible behaviors begins with various pairings of individual transition matrix types: $i$ ) one variety seeker and one reinforcer, ii) both variety seekers, iii) both reinforcers, $i v$ ) one person preferring item $A$ and the other preferring item $B$. The individual transition matrices are then combined under each of the AM's. For ease of exposition we illustrate this step by examining what we call the 90-10 sublibrary (each household has at least one individual transition matrix consisting of .90's and .10's), shown in table 1.

For the first eight rows, the second and third columns show various pairings of individual matrices. The fourth and fifth columns show the household matrix, $\boldsymbol{P}_{H}$, 
Table 1. The 90-10 sub-library

\begin{tabular}{|c|c|c|c|c|c|c|c|c|c|}
\hline \multirow{4}{*}{$\frac{\text { Row }}{1}$} & \multirow{3}{*}{$\frac{P_{1}}{0.10}$} & \multirow{2}{*}{\multicolumn{3}{|c|}{$P_{2}$}} & \multicolumn{4}{|c|}{$P_{H}^{a}$} & \multirow{3}{*}{$\frac{\text { Type }}{\text { Variety \& }}$} \\
\hline & & & & & \multicolumn{2}{|c|}{ Turn taking } & \multicolumn{2}{|c|}{ Decoupled } & \\
\hline & & 0.90 & 0.90 & 0.10 & 0.50 & $0.50^{9}$ & 0.50 & $\cdot 0.50^{y}$ & \\
\hline & 0.90 & 0.10 & 0.10 & 0.90 & 0.50 & 0.50 & 0.50 & 0.50 & Reinforce \\
\hline \multirow{2}{*}{2} & 0.10 & 0.90 & 0.70 & 0.30 & 0.40 & $0.60^{11}$ & 0.55 & $0.45^{11.9}$ & Variety \& \\
\hline & 0.90 & 0.10 & 0.30 & 0.70 & 0.60 & 0.40 & 0.45 & 0.55 & Reinforce \\
\hline \multirow{2}{*}{3} & 0.30 & 0.70 & 0.90 & 0.10 & 0.60 & $0.40^{10}$ & 0.55 & $0.45^{10.9}$ & Variety \& \\
\hline & 0.70 & 0.30 & 0.10 & 0.90 & 0.40 & 0.60 & 0.45 & 0.55 & Reinforce \\
\hline \multirow{2}{*}{4} & 0.90 & 0.10 & 0.90 & 0.10 & 0.90 & 0.10 & 0.70 & $0.30^{13}$ & Roth Reinforce \\
\hline & 0.10 & 0.90 & 0.10 & 0.90 & 0.10 & 0.90 & 0.30 & 0.70 & DUTI KeIMTOLE \\
\hline \multirow{2}{*}{5} & 0.90 & 0.10 & 0.70 & 0.30 & 0.80 & 0.20 & 0.65 & $0.35^{13.111}$ & Both Reinforce \\
\hline & 0.10 & 0.90 & 0.30 & 0.70 & 0.20 & 0.80 & 0.35 & 0.65 & \\
\hline \multirow{2}{*}{6} & 0.10 & 0.90 & 0.10 & 0.90 & 0.10 & 0.90 & 0.30 & $0.70^{12}$ & Both Seek Variety \\
\hline & 0.90 & 0.10 & 0.90 & 0,10 & 0.90 & 0.10 & 0.70 & 0.30 & \\
\hline \multirow{2}{*}{7} & 0.10 & 0.90 & 0.30 & 0.70 & 0.20 & 0.80 & 0.35 & $0.65^{11.12}$ & Both Seek Varietv \\
\hline & 0.90 & 0.10 & 0.70 & 0.30 & 0.80 & 0.20 & 0.65 & 0.35 & Botn seek varlety \\
\hline \multirow{4}{*}{8} & 0.10 & 0.90 & 0.90 & 0.10 & 0.18 & $0.82^{14}$ & 0.50 & $0.50^{9}$ & Opposing \\
\hline & 0.10 & 0.90 & 0.90 & 0.10 & 0.82 & 0.18 & 0.50 & 0.50 & Preferences \\
\hline & & & & & \multicolumn{4}{|c|}{ Null process for row } & \\
\hline & & & & & Turi & taking & $\mathrm{De}$ & oupled & \\
\hline \multirow[t]{2}{*}{9} & 0.50 & 0.50 & 0.50 & 0.50 & \multirow{2}{*}{\multicolumn{2}{|c|}{1}} & \multirow{2}{*}{\multicolumn{2}{|c|}{$1,8,2,3$}} & Coin Tossing \\
\hline & 0.50 & 0.50 & 0.50 & 0.50 & & & & & \\
\hline \multirow[t]{2}{*}{10} & 0.60 & 0.40 & 0.60 & 0.40 & \multirow{2}{*}{\multicolumn{2}{|c|}{3}} & \multirow{2}{*}{\multicolumn{2}{|c|}{3,5}} & \\
\hline & 0.40 & 0.60 & 0.40 & 0.60 & & & & & \\
\hline 11 & $\begin{array}{l}0.40 \\
0.60\end{array}$ & $\begin{array}{l}0.60 \\
0.40\end{array}$ & $\begin{array}{l}0.40 \\
0.60\end{array}$ & $\begin{array}{l}0.60 \\
0.40\end{array}$ & \multicolumn{2}{|c|}{2} & \multicolumn{2}{|c|}{2,7} & \\
\hline \multirow{2}{*}{12} & 0.30 & 0.70 & 0.30 & 0.70 & & & \multirow{2}{*}{\multicolumn{2}{|c|}{6,7}} & \\
\hline & 0.70 & 0.30 & 0.70 & 0.30 & & & & & \\
\hline \multirow{2}{*}{13} & 0.70 & 0.30 & 0.70 & 0.30 & & & \multirow{4}{*}{\multicolumn{2}{|c|}{4,5}} & \\
\hline & 0.30 & 0.70 & 0.30 & 0.70 & & & & & \\
\hline \multirow[t]{2}{*}{14} & 0.20 & 0.80 & 0.20 & 0.80 & & 8 & & & \\
\hline & 0.80 & 0.20 & 0.80 & 0.20 & & & & & \\
\hline
\end{tabular}

a: The superscripted numbers in these two columns refer to the rows which represent the competing null processes.

that would result for the turn-taking and decoupled-superpositioning AM's, respectively. Entries in the fourth column are a simple average of the first two columns. Fifth column entries were derived with logic analogous to that used on pages $262-3$ of $\mathrm{KMW}$.

In addition to discriminating between the turn-taking and decoupled-superpositioning AM's, we also need to determine whether the household is using just a single transition matrix. For example, is a household with empirical $\boldsymbol{p}_{\boldsymbol{H}}=$ $\left(\begin{array}{ll}0.4 & 0.6 \\ 0.6 & 0.4\end{array}\right)$ using just a single transition matrix, or is the purchase string a result 
of matrices in the second row of table 1 being combined by a turn-taking AM (strict compromise)? Hereafter, we refer to the single matrix generating process which corresponds to the $\boldsymbol{P}_{H}$ of a pair of individuals using a specified AM as its competing null process. For example, the null process for row 1 is the coin-tossing process of row 9 . The last six rows of table 1 represent library approximations of the null process of the first eight. Recall that a household purchasing with a single matrix is indistinguishable from one where each member takes turns, but both have the same transition matrix. For each of the first eight rows, the superscripted numbers in columns 4 and 5 refer to the row representing the competing null process. For example, consider row 4 where both individuals have the same transition matrix. Under turn-taking it is neither possible (nor important) to determine whether household members are actually taking turns, or merely behaving as a single entity. However, under decoupled-superpositioning $\boldsymbol{P}_{H}=\left(\begin{array}{cc}0.7 & 0.3 \\ 0.3 & 0.7\end{array}\right)$, and the superscript shows that the competing null process is given by row 13. Thus, the $90-10$ sub-library consists of 22 different data generating processes ( 2 each for the first eight rows, and 1 each for the last six rows).

Step 2: Calculate the true distribution of runs for each hypothesized data generating process.

As stated earlier, each data generating process is hypothesized to be the result of combining pairs of individual matrices with an AM (as in table 1). Each data generating process will then result in a distribution of run lengths. Deriving these distributions is not analytically tractable. Instead, simulation provides a viable path for constructing the true distributions.

Step 3: For a given string of observed purchases, construct the observed distribution of runs, and compute the chi-square goodness of fit statistic for the difference from each true distribution of step 2.

Of course, the observed distribution of run lengths is limited by the length of the panel string. It is impossible to have a run length of 30 in a string length of 20 . In our work so far, we have found that classifying runs as being of lengths one, two, and three or more is sufficient. Finer distinctions of run lengths do not add to the ability to discriminate among competing data generating processes.

Step 4: Assign the observed string to the true distribution for which the chi-square statistic is the minimum. ${ }^{2}$

The approach advocated here is not a standard test of parameter estimates. The ratio of parameters in our models to observations useful for estimating those parameters (i.e. the empirical distribution of runs) renders standard parameter estimates impossible. More than two household members or brands would exponen- 
tially exacerbate this problem. Kerr, Stasser and Davis (1979) distinguish between model fitting and model testing approaches. the latter, useful in research with sparse data, compares several completely specified models to reproduce the relevant data. This is the approach adopted here.

\section{Simulation results}

We performed a simulation study to test the reliability of the procedures outlined above for correctly classifying a string of simulated purchases with its known data generating process. The true distribution of runs was obtained by simulating a sequence of 10,001 purchases. Then, for each of these processes 300 observed purchase strings of lengths $21,5 I$ and $10 I$ were generated. Each of the observed distributions was then classified as belonging to one of the true processes on the basis of the lowest chi-square. For each combination of observed string length and data generating processes, the proportion of times each observed string was classified as belonging to each of the true distributions was then calculated. Instead of presenting the complete set of results (available from the authors) we summarize the conclusions for the 90-10 sub-library. The conclusions drawn from this subset are equivalent to those from examining the entire set of results.

The results (table 2) showed that the turn-taking processes were virtually always classified correctly. The modal classification for the decoupled-superpositioning processes were also correct. In fact, except for the opposing preferences cases, the hit rates were above $60 \%$ even for the shortest string lengths. However, under decoupled-superpositioning and opposing preferences, the observed strings were often misclassified as resulting from coin-tossing $(25.8 \%)$, or decoupled-superpositioning variety $\&$ reinforce $(21.4 \%)$. A similar pattern of misclassification was seen for the observed strings generated according to the coin-tossing process. Recall that under decoupled-superpositioning, the next person to make a purchase is determined randomly. Consequently, when the two individuals have strictly opposing preferences, the household's probabilities of purchasing either brand or the next purchase occasion are equal. This is also true under coin-tossing. Thus, the mutual misclassification between these two processes is to be expected. The misclassification of these two random processes with variety $\&$ reinforce implies that the test is likely to indicate the presence of first-order behavior more often than it should. The results for shorter string lengths were similar in nature, although there was progressive degradation.

The 70-30 sub-library was constructed in a manner similar to the 90-10 library. $\boldsymbol{P}_{1}$ and $\boldsymbol{P}_{2}$ in table 1 were changed as follows: 0.9 and 0.1 were replaced with 0.7 and 0.3 respectively, and 0.7 and 0.3 were replaced with 0.6 and 0.4 respectively. As in the 90-10 sub-library, misclassification across the AM's is uncommon. However, because the switching probabilities of the individual matrices are closer to those of the coin-toss process the results, as expected, were more ambiguous within an AM. 
The following relevant points emerge. First, the more systematic the data generating process is, the more reliably the proposed test classifies it. Second, recall that decoupled-superpositioning is the least predictable version of turn-taking. Consequently, the ability to distinguish it from the corresponding null processes implies that it should also be possible to discriminate any other AM that is more predictable than decoupled-superpositioning from its null process. Taken together, our results imply that if the household's behavior is systematically different from simple compromising, the proposed test can be expected to detect it. This is precisely where relying on transition matrices alone often leads to conclusions of "indifference to variety" (Givon 1984).

\section{Illustrative empirical results}

To illustrate the use of the proposed test we applied it to panel data from the powdered drink mix category. We chose 15 households from a sample of 79 because they were not overwhelmingly loyal to a single brand and had long enough purchase strings (greater than 35). As is customary, the brand with the highest share of a household's purchases was labeled $A$, and the others were grouped into a single other brand $B$. Observed distributions of run lengths were then calculated for each of these households. Classifications were made on the basis of the minimum of the chi-square values computed against each of 156 possible true distributions.

Table 3 presents the results for the households classified as turn-takers. For each household, the first six columns show the household's ID, the purchase string's length, the observed household transition matrix $\left(\boldsymbol{P}_{H}\right)$, the recovered, best-fitting individual transition matrices $\left(\boldsymbol{P}_{1}\right.$ and $\left.\boldsymbol{P}_{2}\right)$, and their types. The last column of table 3 is calculated in the following manner. First, the observed distribution is compared to the true distribution of the null process implied by the observed household transition matrix, $\boldsymbol{P}_{H}$, resulting in a chi-square value for the null process. This chi-square value is then compared to the chi-square value for the best fitting true distribution (upper number). Large values for the ratio reported in the last column (lower number) of table 3 imply that the observed distribution is much more like the best fitting distribution and is unlikely to have been generated by the null process.

Consider now, the first six ID's. For each of these households the best fitting distribution represents a process where one member is variety seeking, the other is reinforcing, and the AM is turn-taking. It is interesting to note that ID's 12, 45, 89 and 99 have very similar household transition matrices, yet the test detects differences in the individual matrices. This reinforces our earlier observation that the same household level transition matrix can arise from very different underlying processes. Note also, that the observed household transition matrix, $\boldsymbol{P}_{H}$, is almost a simple average of the two individual matrices recovered by the test. 


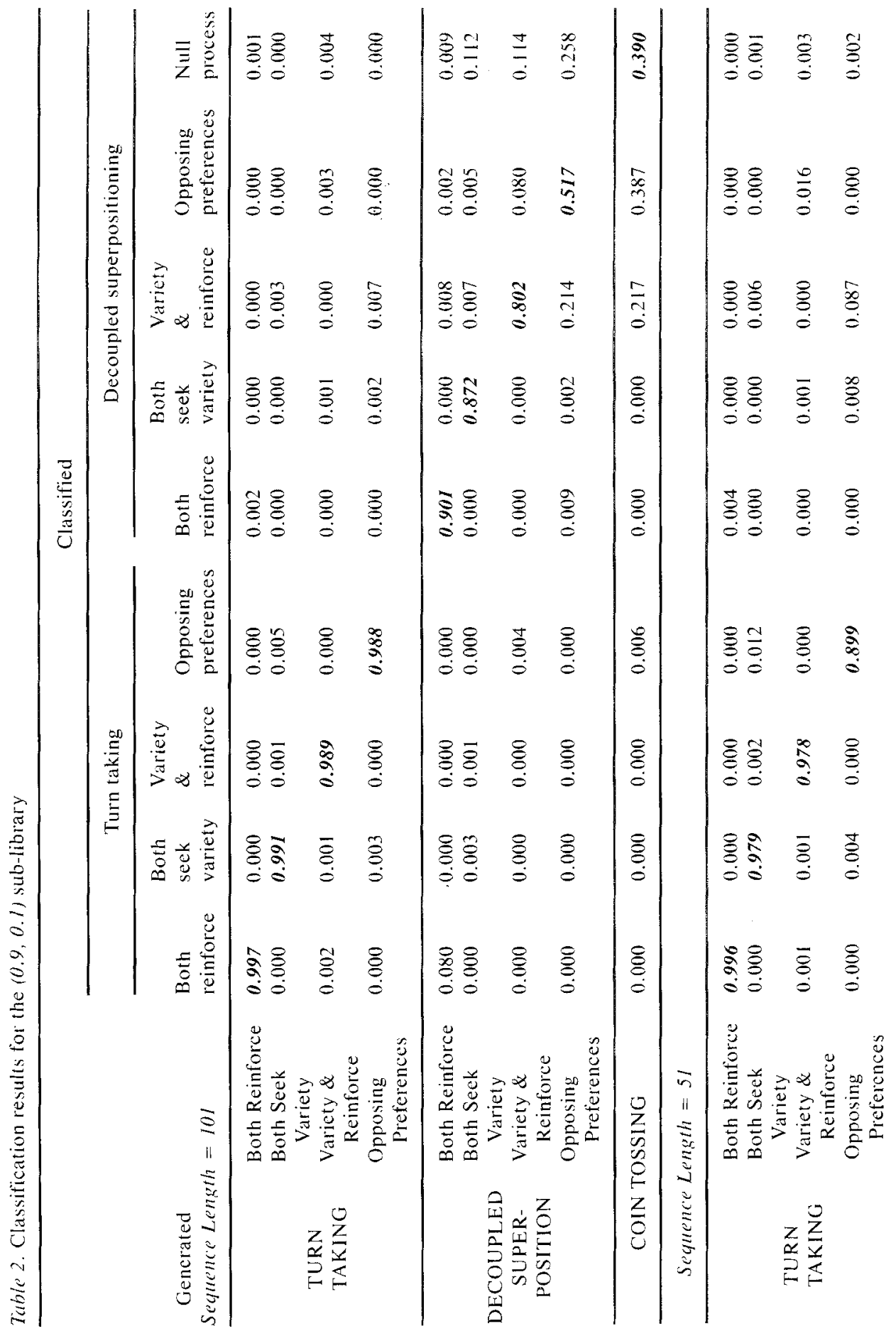




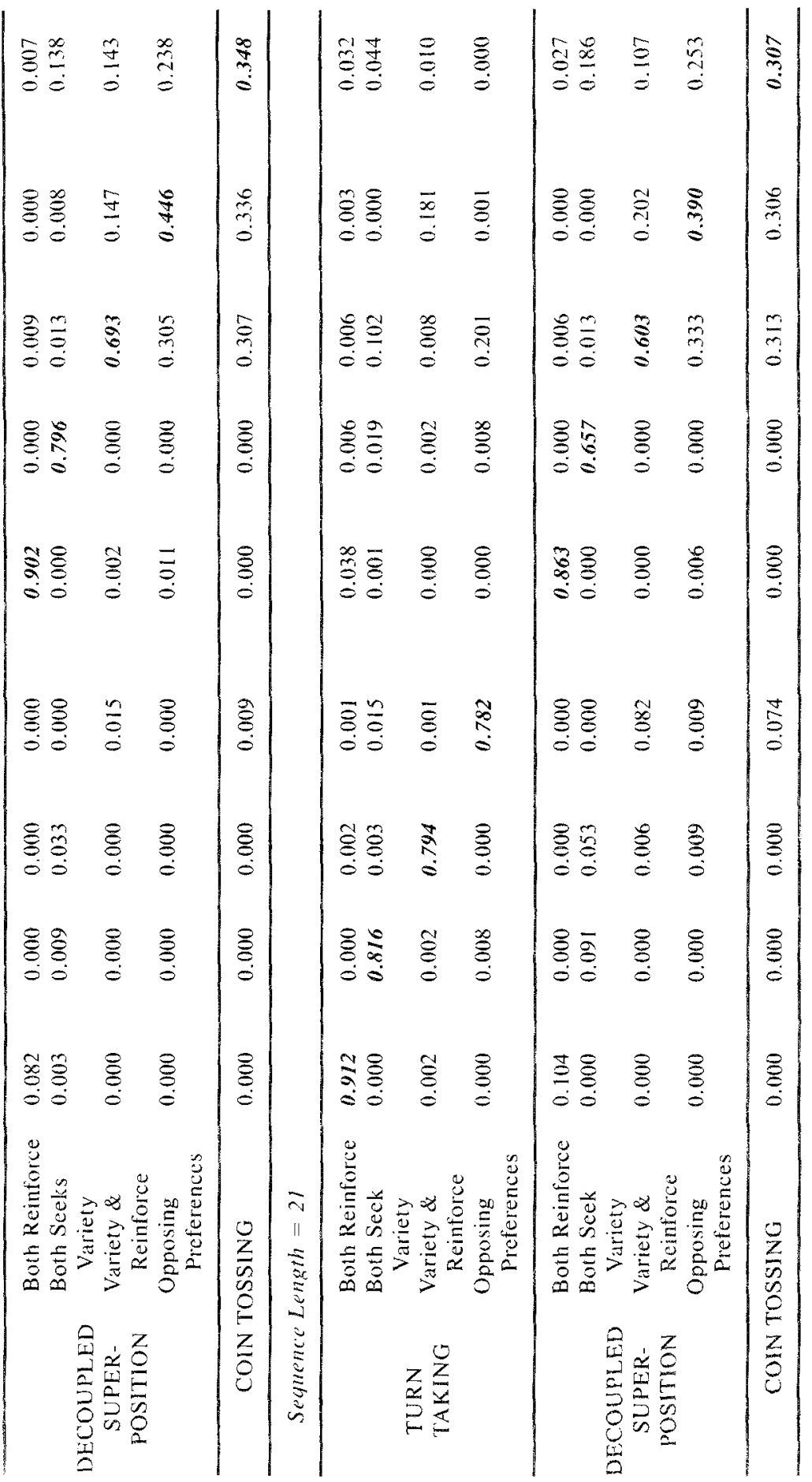


Table 3. Households classified as turn takers

\begin{tabular}{|c|c|c|c|c|c|c|c|c|c|c|}
\hline \multirow{3}{*}{$\frac{\text { ID }}{12}$} & \multirow{3}{*}{$\begin{array}{c}\begin{array}{c}\text { String } \\
\text { length }\end{array} \\
38\end{array}$} & \multicolumn{2}{|c|}{$\mathbf{P}_{\mathrm{H}}$} & \multicolumn{2}{|c|}{$\mathbf{P}_{1}$} & \multicolumn{2}{|c|}{$\mathbf{P}_{2}$} & \multirow{3}{*}{ 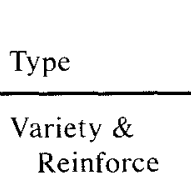 } & \multicolumn{2}{|c|}{$\mathrm{X}_{\text {hest fitling }}^{2}$ ratio } \\
\hline & & 0.63 & 0.37 & 0.50 & 0.50 & 0.80 & 0.20 & & 0.50 & \\
\hline & & 0.39 & 0.61 & 0.50 & 0.50 & 0.20 & 0.80 & & & $18.31 \%$ \\
\hline \multirow{2}{*}{45} & \multirow{2}{*}{63} & 0.64 & 0.36 & 0.40 & 0.60 & 0.90 & 0.10 & Variety \& & 0.34 & \\
\hline & & 0.38 & 0.62 & 0.60 & 0.40 & 0.10 & 0.90 & Reinforce & & $34.72 *$ \\
\hline \multirow{2}{*}{71} & \multirow{2}{*}{87} & 0.52 & 0.48 & 0.30 & 0.70 & 0.80 & 0.20 & Variety \& & 0.74 & \\
\hline & & 0.43 & 0.57 & 0.70 & 0.30 & 0.20 & 0.80 & Reinforce & & $5.88 *$ \\
\hline \multirow{2}{*}{89} & \multirow{2}{*}{67} & 0.63 & 0.37 & 0.40 & 0.60 & 0.90 & 0.10 & Variety \& & 0.46 & \\
\hline & & 0.35 & 0.65 & 0.60 & 0.40 & 0.10 & 0.90 & Reinforce & & $17.70^{*}$ \\
\hline \multirow{2}{*}{99} & \multirow{2}{*}{9.} & 0.65 & 0.35 & 0.40 & 0.60 & 0.70 & 0.30 & Variety \& & 0.19 & \\
\hline & & 0.54 & 0.46 & 0.60 & 0.40 & 0.30 & 0.70 & Reinforce & & $18.85^{*}$ \\
\hline \multirow{2}{*}{128} & \multirow{2}{*}{218} & 0.57 & 0.43 & 0.40 & 0.60 & 0.80 & 0.20 & Variety \& & 0.31 & \\
\hline & & 0.36 & 0.64 & 0.60 & 0.40 & 0.20 & 0.80 & Reinforce & \multirow{3}{*}{0.13} & $17.40^{*}$ \\
\hline \multirow{2}{*}{53} & \multirow{2}{*}{96} & 0.40 & 0.60 & 0.40 & 0.60 & 0.60 & 0.40 & Variety \& & & \\
\hline & & 0.42 & 0.58 & 0.60 & 0.40 & 0.40 & 0.60 & Reinforce & & $3.53^{*}$ \\
\hline & \multirow{2}{*}{\multicolumn{3}{|c|}{$\begin{array}{c}\text { or Decoupled } \\
\text { Superpositioning }\end{array}$}} & 0.80 & 0.20 & 0.10 & 0.90 & Oppos & \multirow{2}{*}{\multicolumn{2}{|c|}{$3.42^{*}$}} \\
\hline & & & & 0.80 & 0.20 & 0.10 & 0.90 & Preferences & & \\
\hline \multirow{4}{*}{83} & \multirow{2}{*}{45} & 0.44 & 0.56 & 0.40 & 0.60 & 0.60 & 0.40 & Variety \& & \multirow[t]{2}{*}{1.00} & \\
\hline & & 0.39 & 0.61 & 0.60 & 0.40 & 0.40 & 0.60 & Reinforce & & $14.30 *$ \\
\hline & \multirow{2}{*}{\multicolumn{3}{|c|}{$\begin{array}{c}\text { or Decoupled } \\
\text { Superpositioning }\end{array}$}} & 0.10 & 0.90 & 0.80 & 0.20 & Opposing & \multirow{2}{*}{\multicolumn{2}{|c|}{$9.97^{\#}$}} \\
\hline & & & & 0.10 & 0.90 & 0.80 & 0.20 & Preferences & & \\
\hline \multirow{2}{*}{184} & \multirow{2}{*}{45} & 0.48 & 0.52 & 0.40 & 0.60 & 0.60 & 0.40 & Opposing & \multirow[t]{2}{*}{0.33} & \\
\hline & & 0.52 & 0.48 & 0.40 & 0.60 & 0.60 & 0.40 & Preferences & & $36.01 *$ \\
\hline 78 & 51 & 0.36 & 0.64 & 0.20 & 0.80 & 0.60 & 0.40 & Opposing & 0.23 & \\
\hline 78 & 56 & 0.54 & 0.46 & 0.20 & 0.80 & 0.60 & 0.40 & Preferences & & $38.57^{*}$ \\
\hline & or D & lied & & 0.30 & 0.70 & 0.30 & 0.70 & Both Seek & $10.76^{\prime}$ & \\
\hline & Superpo & tioning & & 0.70 & 0.30 & 0.70 & 0.30 & Variety & & \\
\hline
\end{tabular}

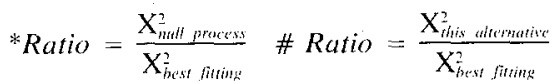

For ID's 53 and 83 the household transition matrix indicates a slight preference for $B$, but the average of the two individual matrices would imply a slight tendency for reinforcement. So, for these two cases the second best-fitting alternatives were also examined. In both cases, the second best process was decoupled-superpositioning with opposing preferences. In table 3 , the last column in the corresponding rows gives the ratio of second best chi-square to the best-fitting chi-square. Finally, households 184 and 78 are also turn-takers, but with opposing preferences. For ID $184, \boldsymbol{P}_{H}$ essentially represents a coin tossing process. But the chi-square for coin-tossing is considerably larger than that for opposing preferences. The test picks up on the relatively smaller proportion of runs of length 2 . For ID $78, \boldsymbol{P}_{\boldsymbol{H}}$ 
shows a variety seeking tendency, but the average of the two individual matrices would imply a preference for $B$. A visual examination of the distributions and the ratios of chi-square values favors the best-fitting process, even though the household transition matrix implied by the second-best process is closer to the observed $P_{H}$.

The first part of table 4 shows the results for the three households classified as compromisers. In each of these cases, the test suggests that the null process fits best and $\boldsymbol{P}_{\mathbf{1}}=\boldsymbol{P}_{\mathbf{2}} \approx \boldsymbol{P}_{\boldsymbol{H}}$. Thus, it is not possible to determine whether the household purchases are based on a single matrix (arrived at through a strict compromise), or are based on two identical matrices taking turns. The second row for each household shows the best fitting decoupled-superpositioning matrices and the ratio of its chi-square to that for the null process. High values of this ratio imply that the null process (a strict compromise) describes the data better than decoupled-superpositioning. Finally, while it is difficult to say whether there is a single household process, or two independent processes generating the data, the types of individual matrices are always the same for the two competing processes. Thus from a theory testing or marketing management point of view the implications are constant across processes.

The second part of table 4 shows two households (ID's $25 \& 80$ ) classified as decoupled-superpositioning, both reinforce. The second best processes in both cases are both reinforcing (with somewhat smaller reinforcement probabilities), but they are turn-takers. Though it is unclear that the best fitting process describes the data better, the types of individual matrices for the competing processes are, once again, the same.

\section{Conclusions}

This paper presented a procedure based on the distribution of run lengths in household level panel data that can allow one to make inferences about the preferences and switching habits of the household members as well as the purchase aggregation mechanisms (AM's) of the household. The procedure was tested in a simulation where series of data were generated by a known process and the procedure was applied to recover the generating processes. Finally, using the procedures, we were able to classify the powdered drink purchases of a set of households.

Our analysis shows that

- significant additional insight into a household's purchase behavior is possible through an examination of the distribution of run lengths

- the ability to recover the underlying data generating process is greatest for the most systematic processes 
Table 4. Households classified as compromisers or decoupled superpositioners

\begin{tabular}{|c|c|c|c|c|c|c|c|c|c|c|}
\hline \multicolumn{11}{|c|}{ Compromisers } \\
\hline \multirow{3}{*}{$\frac{\text { ID }}{19}$} & \multirow{3}{*}{$\begin{array}{c}\begin{array}{l}\text { String } \\
\text { length }\end{array} \\
71\end{array}$} & \multicolumn{2}{|c|}{$\mathbf{P}_{\mathrm{H}}$} & \multicolumn{2}{|c|}{$\mathbf{P}_{1}$} & \multicolumn{2}{|c|}{$\mathbf{P}_{2}$} & \multirow{3}{*}{$\frac{\text { Type }}{\begin{array}{c}\text { Both } \\
\text { reinforce }\end{array}}$} & \multicolumn{2}{|l|}{ Ratio } \\
\hline & & 0.59 & 0.41 & 0.65 & 0.35 & 0.65 & 0.35 & & & \\
\hline & & & & & & & 0.65 & & & \\
\hline & or Dec & upled & & 0.80 & 0.20 & 0.80 & 0.20 & Both & & 8.86 \\
\hline & Superp & sitionin & & 0.20 & 0.80 & 0.20 & 0.80 & Reinforce & & \\
\hline \multirow{4}{*}{162} & 82 & 0.70 & 0.30 & 0.70 & 0.30 & 0.70 & 0.30 & Both & & \\
\hline & $8 z$ & 0.30 & 0.70 & 0.30 & 0.70 & 0.30 & 0.70 & Reinforce & & \\
\hline & or Dec & upled & & 0.90 & 0.10 & 0.90 & 0.10 & Both & & 12.26 \\
\hline & Superp & sitionin & & 0.10 & 0.90 & 0.10 & 0.90 & Reinforce & & \\
\hline \multirow{4}{*}{81} & 269 & 0.39 & 0.61 & 0.40 & 0.60 & 0.40 & 0.40 & Both Seek & & \\
\hline & 209 & 0.55 & 0.45 & 0.60 & 0.40 & 0.60 & 0.60 & Variety & & \\
\hline & or Dec & upled & & 0.30 & 0.70 & 0.30 & 0.70 & Both Seek & & 1.67 \\
\hline & Superp & sitionin & & 0.70 & 0.30 & 0.70 & 0.30 & Variety & & \\
\hline \multicolumn{11}{|c|}{ Decoupled superpositioning } \\
\hline \multirow{2}{*}{25} & \multirow{2}{*}{46} & 0.58 & 0.42 & 0.65 & 0.35 & 0.65 & 0.35 & \multirow{2}{*}{$\begin{array}{l}\text { Both } \\
\text { Reinforce }\end{array}$} & & \\
\hline & & 0.43 & 0.57 & 0.35 & 0.65 & 0.35 & 0.65 & & & \\
\hline \multirow{4}{*}{80} & \multirow{2}{*}{\multicolumn{3}{|c|}{ or Compromise }} & 0.60 & 0.40 & 0.60 & 0.40 & Both & $1.23^{2}$ & \\
\hline & & & & 0.40 & 0.60 & 0.40 & 0.60 & Reinforce & & \\
\hline & \multirow{2}{*}{59} & 0.56 & 0.44 & 0.70 & 0.30 & 0.70 & 0.30 & \multirow{2}{*}{$\begin{array}{l}\text { Both } \\
\text { Reinforce }\end{array}$} & & \\
\hline & & 0.39 & 0.61 & 0.30 & 0.70 & 0.30 & 0.70 & & & \\
\hline & \multirow{2}{*}{\multicolumn{3}{|c|}{ or Compromise }} & 0.60 & 0.40 & 0.60 & 0.40 & \multirow{2}{*}{$\begin{array}{l}\text { Both } \\
\text { Reinforce }\end{array}$} & \multirow[t]{2}{*}{$3.47^{2}$} & \\
\hline & & & & 0.40 & 0.60 & 0.40 & 0.60 & & & \\
\hline
\end{tabular}

- in many instances it should be possible to distinguish, from its null process, any AM where households take turns more predictably than under decoupled-superpositioning

- the ability to recover the data generating process degrades with shorter run lengths

While our results are generally encouraging, several caveats apply. First, the procedures cannot be applied without the use of computer simulations to assess the true distribution of each possible process. Second, selecting the possible be- 
haviors for a given household is a difficult issue. For example, we chose three specific AM's. Clearly others are possible. Crossing these with the various combinations of individual matrices leads, very soon, to a large number of possible behaviors. Staying with just three AM's, our library had 156 possible behaviors. This problem would be further aggravated when considering more than just two brands to choose from. Clearly, the researcher needs to make a judgment based on parsimony, practical utility, and statistical reliability. Third, our paper examines a particular model of household decision making, equation 1. Clearly, other processes such as those discussed by Bawa (1990), and Kahn, Kalwani and Morrison (1986) could also result in distributions of run lengths similar to those generated in our simulation. Future research should examine the efficacy of using a combination of the approaches to gain even sharper insights into the household's behavior and preferences.

\section{Notes}

1. We thank an anonymous reviewer for this suggestion.

2. In our simulation, classifications were also made on the basis of the minimum value of the Kolmogorov-Smirnoff $D$-statistic, and were found to be no more accurate than those based on the chi-square.

\section{References}

Bass, Frank M., Moshe M. Givon, Manohar U. Kalwani, David Reibstein, and Gordon P. Wright. (1984). "An Investigation into the Order of the Brand Choice Process," Marketing Science 3 (Fall), 267-287.

Bawa, Kapil. (1990). "Modeling Inertia and Variety Seeking Tendencies in Brand Choice Behavior," Marketing Science 9 (Summer), 263-278.

Corfman, Kim P., and Sunil Gupta. (1993). "Mathematical Models of Group Choice and Negotiations," In Jehoshua Eliashberg and Gary L. Lillien (eds.), Handbook in ORGMS: Marketing. Boston: Kluwer, 81-140.

Corfman, Kim P., and Donald R. Lehmann. (1987). "Models of Cooperative Group Decision-Making: An Experimental Investigation of Family Purchase Decisions," Journal of Consumer Research 14 (June), 1-13.

Corfman, Kim P., Donald R. Lehmann, and Joel H. Steckel. (1990). "Longitudinal Patterns of Group Decisions: An Exploratory Analysis," Multivariate Behavioral Research 25 (July), 249-273.

Frank, Ronald E. (1962). "Brand Choice as a Probability Process," Journal of Business 35 (January), 43-56.

Givon, Moshe M. (1984). "Variety Seeking through Brand Switching," Marketing Science 3 (Winter), $1-22$.

Jeuland, Abel P., Frank M. Bass, and Gordon P. Wright. (1980). "A Multibrand Stochastic Model Compounding Heterogenous Erlang Timing and Multinomial Choice Process," Operations Research 28 (March-April), 255-277.

Kahn, Barbara E., Manohar U. Kalwani, and Donald G. Morrison. (1986). "Measuring Variety Seeking and Reinforcement Behaviors using Panel Data," Journal of Marketing Research 23 (May), 89-100. 
Kahn, Barbara E., Donald G. Morrison, and Gordon P. Wright. (1986). "Aggregating Individual Purchases to the Household Level," Marketing Science 5 (Summer), 260-268.

Kalwani, Manohar U., and Donald G. Morrison. (1977). "A Parsimonious Description of the Hendry System," Management Science 23 (January), 467-477.

Kerr, Norman L., Garold Stasser, and James H. Davis. (1979). "Model Testing, Model Fitting, and Social Decision Schemes," Organizational Behavior and Human Performance 23, 399-410.

Lehmann, Donald R. (1972). "Judged Similarity and Brand-Switching Data as Similarity Measures," Journal of Marketing Research 9 (August), 331-334.

Massy, William F., David B. Montgomery, and Donald G. Morrison. (1970). Stochastic Models of Buying Behavior. Cambridge, MA: MIT Press.

Steckel, Joel H., Kim P. Corfman, David J. Curry, Sunil Gupta, and James Shanteau. (1991). "Prospects and Problems in Group Decisions," Marketing Letters 2 (August), 231-240.

Steckel, Joel H., Donald R. Lehmann, and Kim P. Corfman. (1988). "Estimating Probabilistic Choice Models from Sparse Data: A Method and an Application to Groups," Psychological Bulletin 103 (January), 131-139. 